\title{
Control the tightness of the pipeline valve shutter according to the change in the Hurst exponent of vibroacoustic signals
}

\author{
A. R. Zagretdinov ${ }^{1, *}$, R. B. Kazakov ${ }^{1}$, and A. A. Mukatdarov ${ }^{1}$ \\ ${ }^{1}$ Kazan State Power Engineering University, Kazan, Russia
}

\begin{abstract}
The article is devoted to the study of the possibility of applying the method of standardized scope ( $\mathrm{R} / \mathrm{S}$ analysis) to control the tightness of the pipeline valve shutter. Experimental studies have confirmed the possibility of using R/S analysis to control the tightness of the pipeline valve shutter. Hurst's exponent allows you to qualitatively determine the flow of the pipeline valve shutter.
\end{abstract}

\section{Introduction}

Pipeline valves are an indispensable element of most technological systems. It provides the implementation of a set of functions, the reliable execution of which contributes to the continuity of technological processes in the distribution, regulation and discharge of working substances.

The classification of valve is very diverse. It emphasizes the importance and diversity of the tasks solved by it. By function, pipeline valves can be divided into:

- shut-off (including drain (drainage) and control);

- regulating (including reducing (throttle) and shutoff and regulating);

- protective (tripping, cut-off, including check valves);

- safety;

- distribution and mixing;

- phase separation (including condensate traps).

Pipeline valve is widely used in such energyintensive areas as housing and utilities, heat and power (including nuclear), oil and gas and chemical industry, metallurgy, etc. Improper functioning of valve results in lower energy efficiency and safety of technological processes.

A critical defect of pipeline valve is a violation of its tightness.

Leakage of the valve occurring in the external environment can be detected during an external inspection following the traces of the corpus frostbite, drips, noise and gas pollution near the valve. However, leakages in the valve shutter do not have external signs, and require the use of special methods of control. Such methods include hydro or pneumatic tests with pressure measurement before and after the valve shutter. Such tests are rather laborious and not suitable for operational control.

The acoustic leak-detection method [1,2] is widely used as an express method for control the tightness of valves shutter. It is based on the indication of acoustic oscillations excited in the object when a liquid or gas leaks through the leakiness. The pass of the working environment is detected by the increase in the amplitude of the diagnostic signal (during spectral processing - the amplitudes of the harmonics). In the case when the amplitude does not increase due to leakage, but due to changes in other parameters of the system, a false signal is generated.

Thus, the use of new methods for control the tightness of pipeline valve shutter, which react not to an increase in amplitude, but to a change in the signal structure, is relevant.

\section{Methods of study}

The small size of the leaks makes the flow turbulent, even with small pressure drops. In a turbulent flow, chaotic fluctuations of speed and pressure are always present, which are created by numerous vortices of various sizes. As a result, when the working substance passes through the shutter, vibrational oscillations of a complex structure are excited on the corpus of pipeline valve. The analysis of valve vibration using the methods of the deterministic chaos theory will allow to increase the reliability of the control of the working substance pass.

For a quantitative assessment of the randomness of series, the method of normalized scope (R/S analysis), proposed by the British hydrologist G.E. Hurst.

The basis of the statistical model of Hurst was the work of A. Einstein on Brownian motion, which is essentially a model of random walk of a particle. The essence of the theory is that the distance $R$ that a particle travels, increases in proportion to the square root of time $T$ :

$$
R=T^{0,5}
$$

\footnotetext{
*Corresponding author: azagretdinov@yandex.ru
} 
Hurst expanded Einstein's equation and led him to a more general form:

$$
\frac{R}{S}=c \cdot n^{H}
$$

where $R$ is the normalized range of variation, $S$ is the standard deviation, $c$ is a constant, $n$ is the number of sample elements, $H$ is the Hurst exponent.

If the process is random, then the Hurst exponent $H=$ 0.5 . When $H=0.5 \div 1$, the normalized range changes faster than the square root of time, i.e. the system travels more than the probabilistic process. This process is called persistent. $H=0 \div 0.5$ means an antipersistent process. In this case, the system is characterized by frequent, but small changes. [3-7]

B. Mandelbrot, the creator of fractal geometry, discovered laws in the Hurst formula that are related to the fractality of time series. The Hurst exponent is related to the Hausdorff-Besikovich dimension by the following relation [8-10]:

$$
D=2-H
$$

\subsection{R/S analysis algorithm}

Let there is a sample of $n$ elements, which is a time series. The algorithm for the implementation of the $\mathrm{R} / \mathrm{S}$ analysis is as follows [3].

1. The smallest own divisor $m$ of the sample $n$ is determined. Sample $n$ is divided by $k=n / m$ groups.

Obviously, the number of elements in each group is $m$. Elements in each group are denoted by $t_{i}$.

2. For each group, an average value is calculated

$$
\overline{t_{k}}=\frac{1}{m} \sum_{i=1}^{m} t_{i}, \frac{1}{m} \sum_{i=m+1}^{2 m} t_{i} \ldots \frac{1}{m} \sum_{i=(k-1) m+1}^{n} t_{i}
$$

3. For each group rescaled range is calculated

$$
R_{k}=\max \left(X_{i}\right)-\min \left(X_{i}\right)
$$

4. For each group the average deviation is calculated $S_{k}$ according to the standard formula

$$
S_{k}=\sqrt{\frac{1}{m} \sum_{i=1}^{m}\left(t_{i}-\overline{t_{k}}\right)^{2}}
$$

5. For each group $R / S$ index is determined as $R_{k} / S_{k}$. Then the average range of variation is found

$$
\overline{R / S_{j}}=\frac{1}{k} \sum_{i=1}^{k} R / S_{i}
$$

Index $j$ in this case means that the average range of variation is obtained at the $j$-th step, which corresponds to the $j$-th own divider.

6 . The procedure described above is repeated, using as $m$ all possible own dividers. In the last step $m=n / 2$.

Thus, the sample $\overline{R / S_{j}}$ is obtained.
The number of elements in the sample corresponds to the number of own dividers.

7. Dependencies graph $\log R / S$ from $\log m$ is under construction and using the least squares method is the regression equation of the form

$$
\log R / S=H \cdot \log m+\log c
$$

where $H$ - Hurst exponent.

The most common result of the $\mathrm{R} / \mathrm{S}$ analysis is a curved line, therefore, it is advisable to determine the Hurst exponent at window length intervals $m$ [11].

\subsection{Experimental studies}

In order to determine the possibility of using $\mathrm{R} / \mathrm{S}$ analysis for vibroacoustic control the tightness of the pipeline valve shutter, there were conducted experimental studies.

The scheme of the experimental stand is shown in Figure 1.

The opening of the valve 1 regulated the consumption of circulating water. The dependence of the throughput capacity of the valve on the degree of valve closure has a nonlinear characteristic. The flow characteristic of the valve is influenced by all elements of the hydraulic system. Therefore, the flow through the valve was monitored using a meter 3 .

Vibration acceleration sensor 6 was attached to pipeline 2 in close proximity to valve 1 . For recording and analysing signals, an original software package was used, written in LabView [12-14]. The analog-to-digital converter sampling frequency is taken equal $44.1 \mathrm{kHz}$. Signals with a length of 10000 samples were analysed, the Hurst exponent was calculated over the interval log $m=3.32 \div 8$.

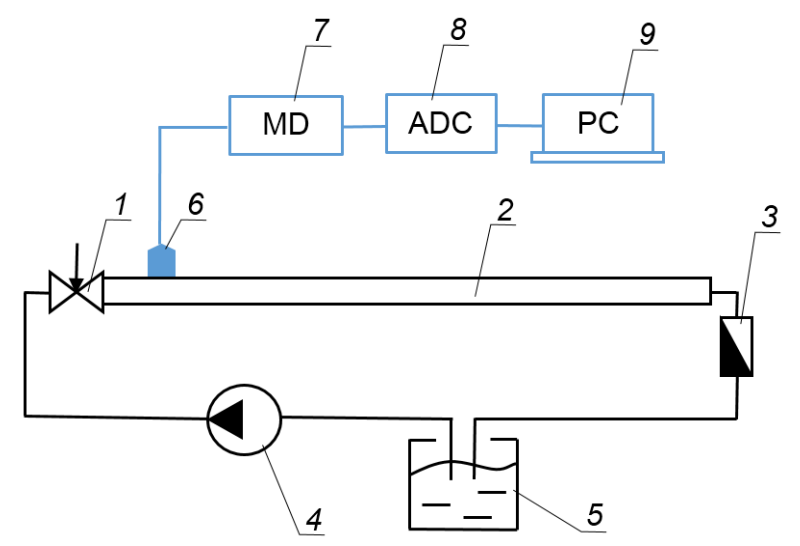

Fig. 1. Experimental stand: 1 - valve, 2 - pipeline; 3 - meter; 4 water capacity; 5 - pump; 6 - vibration acceleration sensor AP2038P; 7 - matching device AG01-3; 8 - analog-to-digital converter NI USB-6229; 9 - computer with installed software package.

\section{Results}

The characteristic vibroacoustic signals recorded when the valve is opened and the Hurst dependence graphs for them are shown in Figures 2, 3. 


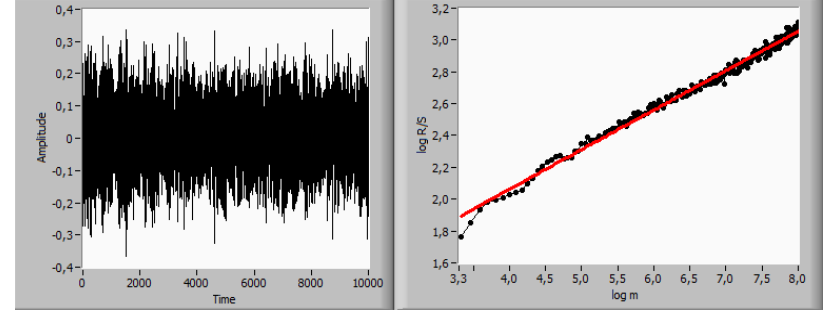

Fig. 2. Vibroacoustic signal and the Hurst dependence at a water consumption of $4 \mathrm{l} / \mathrm{min}$ (red line - regression graph).

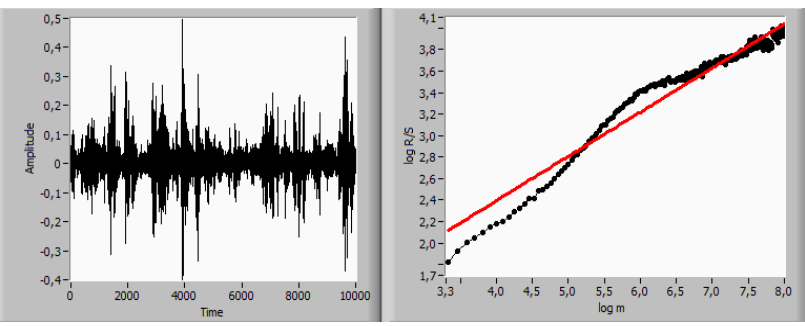

Fig. 3. Vibroacoustic signal and the Hurst dependence graph at a water consumption of $25 \mathrm{l} / \mathrm{min}$ (red line - regression graph).

The dependence of the Hurst exponent on the flow rate of water passing through the valve is shown in Figure 4 . The consumption of $35 \mathrm{l} / \mathrm{min}$ corresponds to the water consumption with the valve fully open. Vibroacoustic signals at consumptions of 0 and $35 \mathrm{l} / \mathrm{min}$ are persistent, for them the Hurst exponent is higher than the level of the probabilistic process $(H>0.5)$. At lower consumptions than $35 \mathrm{l} / \mathrm{min}$, the Hurst exponent decreases, the oscillatory process becomes antipersistent $(H<0.5)$. Antipersistent process is faster than random, and is characteristic of the turbulence effects.

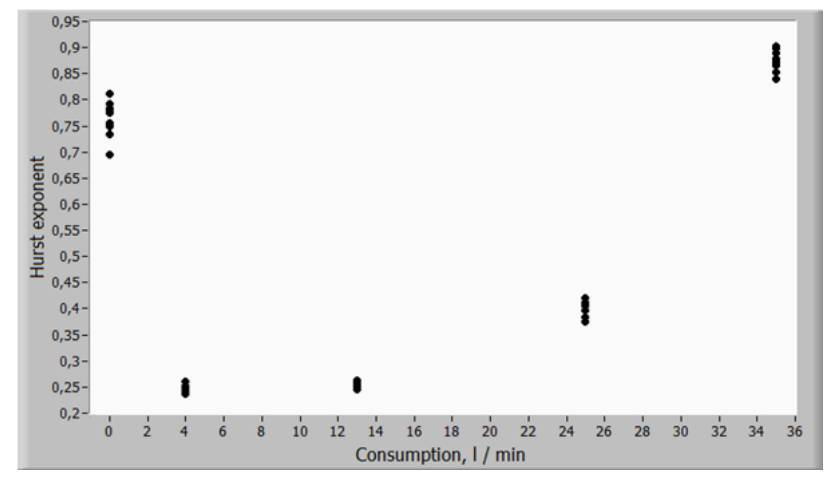

Fig. 4. The dependence of the Hurst exponent on a water consumption.

\section{Conclusions}

During control pipeline valve, as a rule, it is not fundamentally necessary to accurately estimate the leakage consumption, because they are not allowed in most technological processes [15]. Experimental studies have confirmed the possibility of using $\mathrm{R} / \mathrm{S}$ analysis to control the tightness of the pipeline valve shutter. Hurst's exponent allows you to qualitatively determine the flow according to the criterion "yes or no".

\section{References}

[1] A.D. Prituzhalov, N.L. A.V. Kapitonov, Vorontsov, A.M. Kapitonov, A Leakage Detection in Valves during Operation Oil and gas technologies 3(80), 50-3 (2012)

[2] Sh.G. Ziganshin, E.V. Izmailova, A.V. Maryashev, Technique for search of pipeline leakage according to acoustic signals analysis IOP Conference Series: Materials Science and Engineering 86012035 (2017)

[3] H. Hurst, Long term storage capacity of reservoirs Transactions of the American society of Civil Engineers 116, 770-99 (1951)

[4] A.V. Zinenko, R/S analysis in the stock market Business-informatics 3(21), 24-30 (2012)

[5] Yu.A. Kalush, V.M. Loginov, The Hurst index and its hidden properties Siberian journal of industrial mathematics 4(12), 29-37 (2002)

[6] A.P. Lepikhin, D.I. Perepelitsa, To the application of the Hurst index (coefficient) in hydrology Geographical bulletin. Hydrology 4(39), 36-43 (2016)

[7] L.O. Kirichenko, Comparative analysis of the statistical properties of Hurst estimates index Bulletin of NTU «XII». Special topic: Informatics and modelling 21, 88-96 (2010)

[8] B. Mandelbrot, Fractal geometry of nature (Moscow: Publishment of Computer researches institute) 656 (2002)

[9] B. Mandelbrot, Fractals, case and finance (Izhevsk: regular and chaotic dinamics) 255 (2004)

[10] M. Kale, F.B. Butar, Fractal analysis of time series and distribution properties of Hurst exponent Journal of Mathematical Sciences \& Mathematics Education 5(1), 8-19 (2005)

[11] V. Butakov, A. Grakovskiy, Estimation of the stochasticity of time series of arbitrary origin using the Hurst index Computer Modelling and New Technologies 9(2), 27-32 (2005)

[12] I.V. Shvetsov, Yu.V. Vankov, A.R. Zagretdinov, Control of rotary equipment unbalance with using statistical criteria comparison of vibration spectrum IOP Conference Series: Materials Science and Engineering 441(1), 012051 (2018)

[13] E.R. Saifullin, A.R. Zagretdinov, S.G. Ziganshin, Yu.V. Vankov, Control of the rotary equipment disbalance by the spectrum of envelope vibroacoustic signal Journal of Advanced Research in Dynamical and Control Systems 10(2), 2242-7 (2018)

[14] E.R. Saifullin, S.G. Ziganshin, Y.V. Vankov, A.R. Zagretdinov, Assessment of technical condition of polyurethane foam thermal insulation pipelines of heating networks using neural network technologies International Journal of Engineering and Technology (UAE) 7, 241-4 (2018)

[15] M.V. Babenkov, D.V. Kopytov, D.V. Gurevich, M.N. Kuznetsov, Experimental study of acoustic emission parameters of leaks in production tree valves Automation, telemechanization and communication in the oil industry 16, 12-4 (2015) 